"The role of helper lipids in the intracellular disposition and transfection efficiency of niosome formulations for gene delivery to retinal pigment epithelial cells.” Ojeda E., Puras, G., Agirre, M., Zarate, J., Grijalvo, S., Eritja, R., DiGiacomo, L., Caracciolo, G., Pedraz, J.L. Int. J. Pharm., 503(1-2), 115-126 (2016). doi: 10.1016/j.jpharm.2016.02.043

\title{
The role of helper lipids in the intracellular disposition and transfection efficiency of niosome formulations for gene delivery to retinal pigment epithelial cells
}

\author{
Edilberto Ojeda ${ }^{a, b}$, Gustavo Puras ${ }^{a, b}$, Mireia Agirre ${ }^{a, b}$, Jon Zarate ${ }^{a, b}$, Santiago Grijalvo ${ }^{b, c}$, Ramon \\ Eritja $^{b, c}$, Luca DiGiacomo ${ }^{d}$, Giulio Caracciolo ${ }^{d}$, Jose-Luis Pedraz ${ }^{a, b, *}$ \\ ${ }^{a}$ NanoBioCel Group, University of Basque Country (UPV/EHU), Vitoria-Gasteiz, Spain \\ ${ }^{b}$ Biomedical Research Networking Center in Bioengineering, Biomaterials and Nanomedicine \\ (CIBER-BBN), Vitoria-Gasteiz, Spain \\ ${ }^{c}$ Institute of Advanced Chemistry of Catalonia, IQAC-CSIC, Barcelona, Spain \\ 'Department of Molecular Medicine, "Sapienza" University of Rome, Viale Regina Elena, 324, \\ 00161 Rome, Italy
}

Abstract. In this work, we carried out a comparative study of four different niosome formulations based on the same cationic lipid and non-ionic tensoactive. The niosomes prepared by oil-in-water emulsion technique (o/w) only differed in the helper lipid composition: squalene, cholesterol, squalane or no helper lipid. Niosomes and nioplexes elaborated upon the addition of pCMS-EGFP reporter plasmid were characterized in terms of size, zeta potential and polydispersity index. The capacity of the niosomes to condense, release and protect the DNA against enzymatic degradation was evaluated by agarose gel electrophoresis. In vitro experiments were carried out to evaluate transfection efficiency and cell viability in retinal pigment epithelial cells. Moreover, uptake and intracellular trafficking studies were performed to further understand the role of the helper lipids in the transfection process. Interestingly, among all tested formulations, niosomes elaborated with squalene as helper lipid were the most efficient transfecting cells. Such transfection efficiency could be attributed to their higher cellular uptake and the particular entry pathways used, where macropinocytosis pathway and lysosomal release played an important role. Therefore, these results suggest that helper lipid composition is a crucial step to be considered in the design of niosome formulation for retinal gene delivery applications since clearly modulates the cellular uptake, internalization mechanism and consequently, the final transfection efficiency.

Keywords: Niosomes; Non-viral vector; Cationic lipid; Transfection; Helper lipid; Intracellular trafficking. 
Abbreviations: CME, clathrin-mediated endocytosis; CVME, caveolae-mediated endocytosis; Sque, Squalene; Cho, cholesterol; Squa, squalane; None, none helper lipid; ARPE-19, retinal pigment epithelial cells; PDI, polydispersity index; SDS, sodium dodecyl sulfate; SC, supercoiled; OC, open circular; MFI, mean fluorescence intensity; CLSM, Confocal laser scanning microscopy experiments; M1, Mander's overlap coefficient.

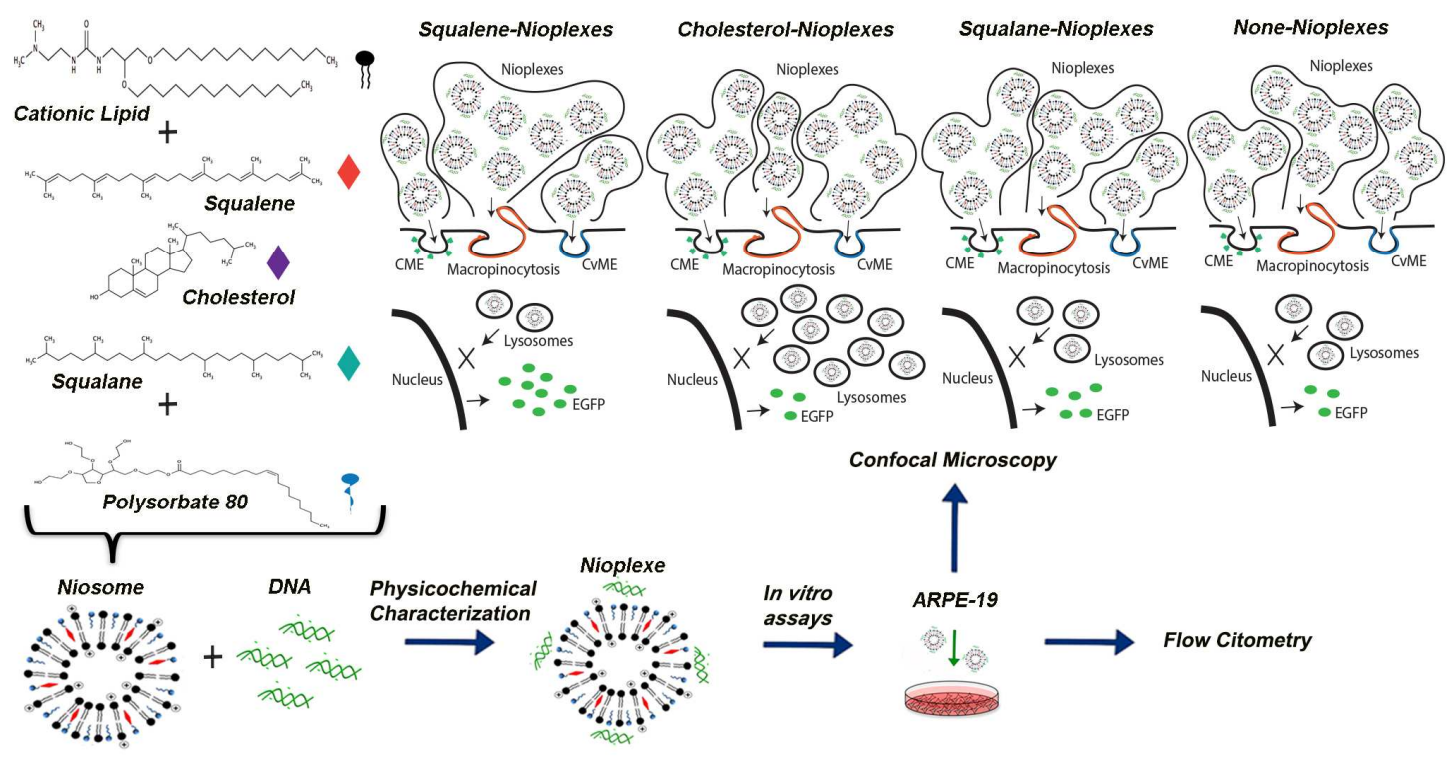

\section{INTRODUCTION}

Gene therapy represents a promising approach that aims to address the disease from the molecular point of view. This type of therapy is based on modified or normal functioning gene copies that are delivered into the cell nucleus to produce bioactive agents (Anderson, 1998). The delivery of naked DNA is an inefficient process due to the negatively charged nature of nucleic acids that difficult the passage through cell membrane and the presence of enzymes that can digest the naked DNA. Therefore, it is necessary the use of capable vectors to deliver efficiently the DNA inside the cells, such as non-viral vectors that are mainly based on cationic polymers and lipids. Compared to viral vectors, non-viral vectors show a reduced persistence of transfection and low transfection efficiencies in cells. On the other hand, non-viral vectors are cheaper, easy to elaborate, and the size of DNA inserted is hypothetically limitless. Moreover, they do not exhibit antigen-specific immune and inflammatory response (Charbel Issa and MacLaren, 2012). Consequently, non-viral vectors have captured the attention of the research community in the last 20 years.

Niosomes are drug carrier systems similar to liposomes with a bilayer structure, where the phospholipids of the liposomes have been substituted by non-ionic surfactants. Compared to liposomes, niosomes show some significant advantages, such as low cost and high chemical and storage stabilities. Even though the application of niosomes in gene therapy has been poorly studied, some optimistic results have been recently reported in the literature that 
highlights the satisfactory properties of niosomes for gene delivery purposes (Ojeda et al., 2015b; Puras et al., 2014).

Niosomes as gene delivery vectors are commonly based on non-ionic surfactants, cationic lipids and helper lipids. Over the years, several researchers have studied these components and their effect on the niosome formulations. Such studies have shown that nonionic surfactants make niosome formulations stable, and prevent the formation of aggregates of the particles (Choi et al., 2004; Huang et al., 2011; Moghassemi and Hadjizadeh, 2014). Cationic lipids handle the interaction with the negatively charged DNA and its condensation to form nioplexes by electrostatic interactions (Karmali and Chaudhuri 2007). Additionally, it has been observed that cationic lipid chemical structures influence on the niosomes charge, toxicity, biodegradability, and transfection efficiencies (Byk et al., 1998; Ojeda et al., 2015b; Zhi et al., 2010). Regarding helper lipids, it has been described that they are responsible for enhancing the physicochemical properties of the emulsion and the improve-ment of gene delivery (Dabkowska et al., 2012; Mochizuki et al., 2013). However, the mechanisms that involve these improvements in cationic niosome formulations for gene delivery applications have not been completely understood, and more detailed studies are required.

The final impact on gene expression, among many other factors, clearly depends on the cell to be transfected and on the capacity of the vector to enter the cell and the posterior pathway employed to deliver its cargo into the nucleus (Agirre et al., 2015). Different endocytic routes can mediate the cellular uptake and the final cargo delivery. Among these endocytic routes clathrin-mediated endocytosis (CME), caveolae-mediated endocytosis (CVME) and macropinocytosis are among the most studied (Cardarelli et al., 2012; Marchini et al., 2010; Zhao et al., 2011). Additionally to the uptake pathways, the particle transport mechanisms can deter-mine the final intracellular fate of the vector, e.g., lysosomal degradation (Pozzi et al., 2014). Such pathways have their particular characteristics and their intervention in the cellular uptake and further internal processing will depend on many factors related with the gene delivery vector such as the size, surface charge, morphology and composition (Luzio et al., 2009; Xiang et al., 2012; Zhao et al., 2011). Therefore, it is necessary to analyze and understand all these factors to develop more efficient non-viral vectors for gene delivery applications.

Thus, we carried out a comparative study of four different niosome formulations based on the same cationic lipid and non-ionic surfactant, but different helper lipid, to determine its role in the niosome formulations and the transfection process mediated by nioplexes upon the addition of pCMS-EGFP reporter plasmid. The niosome formulations only differed in the helper lipid: Squalene (Sque), cholesterol (Cho), squalane (Squa) or none helper lipid (None). Niosomes prepared by oil-in-water emulsion technique $(\mathrm{o} / \mathrm{w})$, and nioplexes were characterized in terms of size, zeta potential and polydispersity index. The capacity of the niosomes to condense, release and protect the DNA against enzymatic degradation was evaluated by agarose gel electrophoresis. In vitro experiments were performed in retinal pigment epithelial cells (ARPE-19) cells by flow cytometry to assess the transfection efficiency of nioplexes and cell viability. Additionally, we carried out cell uptake studies at $1 \mathrm{~h}$ after the addition of the nioplexes. To comprehend the internalization process, we analyzed cell trafficking of our formulations in different entry pathways (CME, CVME, macropinocytosis) and 
lysosomal compartment. Colocalization was analyzed through Mander's overlap coefficient between the nioplexes (niosomes/Cy3 stained DNA) and the above stained endocytic pathways.
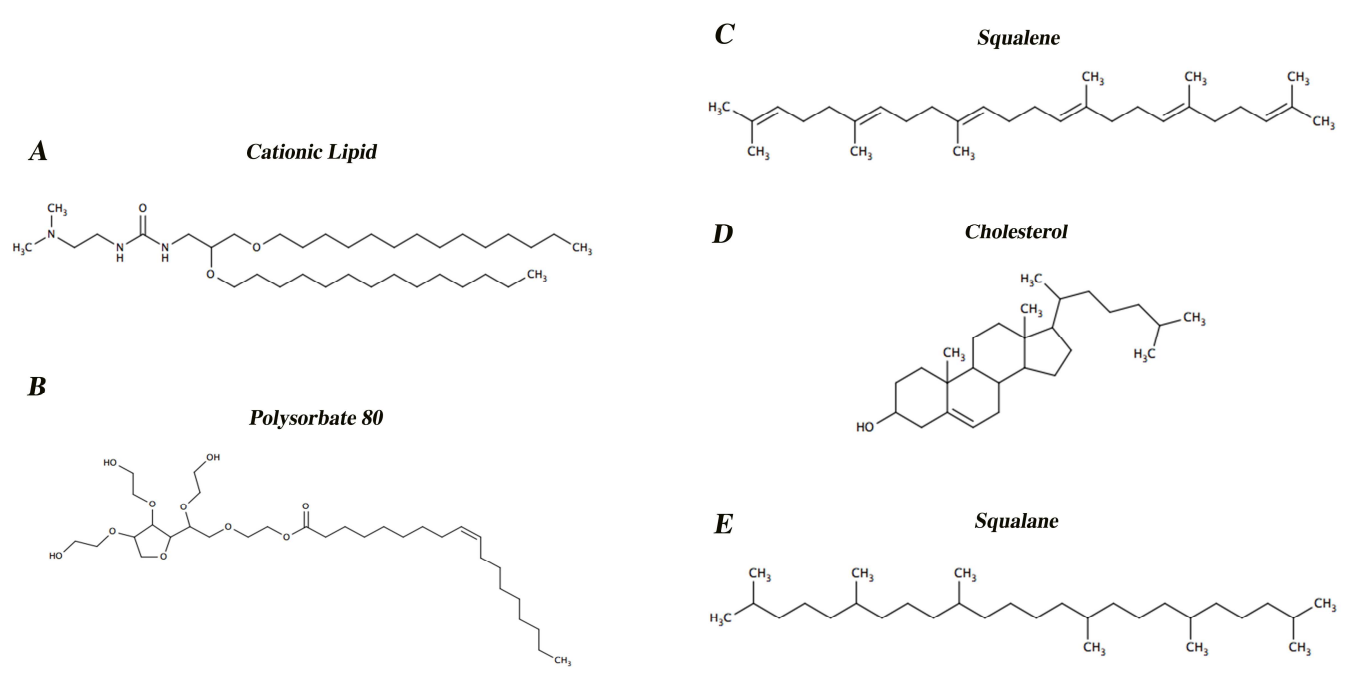

Fig. 1. Chemical structures of niosome components. A) Cationic lipid: 1-(2dimethylaminoethyl)-3-[2,3-di(tetradecyloxy) propyl]urea. B) Polysorbate 80. C) Squalene: (6E,10E,14E,18E)-2,6,10,15,19,23-hexamethyltetracosa-2,6,10,14,18,22-hexaene Cholesterol: 2,15-dimethyl-14-(6-methylheptan-2-yl)tetracyclo[8.7.0.02,7.011,15]heptadec-7en-5-ol. E) Squalane: 2,6,10,15,19,23- hexamethyltetracosane.

\section{Material and methods}

\subsection{Preparation of niosomes.}

Cationic lipid 1-(2-dimethylaminoethyl)-3-[2,3-di(tetradecy- loxy) propyl]urea was synthetized in the laboratory, see supporting information (Fig. SI 1), to elaborate all cationic niosome formulations. Niosomes based on the aforementioned cationic lipid (Fig. 1A), polysorbate 80 (Fig. 1B) (Tween 80, Sigma-Aldrich, Madrid, Spain) and different helper lipids were prepared using the o/w emulsification technique, as previously reported (Ojeda et al., 2015b). We prepared four niosome formulations, where three formulations were prepared with different helper lipids and one formulation without helper lipid (None). Briefly, $5 \mathrm{mg}$ of the cationic lipid were gently grounded with either $23 \mu \mathrm{l}$ of squalene (Sque) (Fig. 1C), $100 \mu \mathrm{l}$ of cholesterol (Cho) (Fig. 1D) or $24.69 \mathrm{ml}$ of squalane (Squa) (Fig. 1E) (Sigma-Aldrich, Madrid, Spain). The concentration of all helper lipids in each formulation was $10 \mathrm{mM}$. Then, $1 \mathrm{ml}$ of dichloromethane (DCM) (Panreac, Barcelona, Spain) was added to all four formulations and emulsified with $5 \mathrm{ml}(0.5 \%, \mathrm{w} / \mathrm{w})$ of the non-ionic surfactant aqueous solution of polysorbate 80. Sonication was used to obtain the emulsion (Branson Sonifier 250, Danbury) for $30 \mathrm{~s}$ at 50 $\mathrm{W}$. The organic solvent was removed from the emulsion by evaporation under magnetic agitation for $3 \mathrm{~h}$ at room temperature inside the extraction hood. Upon DCM evaporation, a 
dispersion containing the nanoparticles was formed by precipitation of the nanoparticles in the aqueous medium. The final cationic lipid concentration obtained was $1 \mathrm{mg} / \mathrm{ml}$.

\subsection{Plasmid propagation and preparation of nioplexes}

Escherichia coli DH5- $\alpha$ was used to propagate the pCMS-EGFP plasmid (PlasmidFactory, Bielefeld, Germany). According to the manufacturer's purification instructions, the plasmid was treated using the Qiagen endotoxin-free plasmid purification Maxi-prep kit (Qiagen, Santa Clarita, CA, USA). The concentration of pDNA was quantified using a NanoDrop1 (ND-1000 spectrophotometer, Thermo Fisher Scientific Inc., Denver, USA) measuring the absorbance at $260 \mathrm{~nm}$. Agarose gel electrophoresis in Tris borate-EDTA buffer, $\mathrm{pH} 8.0$ (TBE buffer) was used to verify the purity of the plasmid. DNA bands were detected using GelRedTM (Biotium, Hayward, California, USA) and images were observed with a ChemiDocTM MP imaging system (Bio-Rad, USA). The nioplexes formed by the addition of DNA to the niosomes and expressed as the ratio of cationic lipid/DNA (w/w) were elaborated by mixing cationic lipid/DNA at 30/1 (w/w) mass ratio. The mixture was left for $30 \mathrm{~min}$ at room temperature to enhance electrostatic interactions between the cationic lipid of the niosomes and the plasmid. The stock solution of plasmid pCMS-EGFP $(0.5 \mathrm{mg} / \mathrm{ml})$ was estimated to be around 0.137 micromolar (pCMS-EGFP, 5541 bp, average MW 3657060). For confocal fluorescence microscopy and uptake cytometry experiments, we used Cy3- labeled $2.7 \mathrm{kbp}$ plasmid DNA (0.5 mg/ml) (Mirus Bio Corporation, Madison, WI, USA).

\subsection{Size, polydispersity index and zeta potential measurements.}

The hydrodynamic diameter and the zeta potential of the niosomes and nioplexes were determined by Dynamic Light Scattering (DLS) and by Lasser Doppler Velocimetry (LDV), respectively. The data were obtained using a Zetasizer Nano ZS (Malvern Instrument, UK) as previously described (Ojeda et al., 2015b). Briefly, $50 \mu \mathrm{l}$ of the niosomes and nioplexes were resuspended into $950 \mu \mathrm{l}$ of $0.1 \mathrm{mM} \mathrm{NaCl}$ solution to determined size and polydispersity index (PDI). All measurements were carried out in triplicate. The particle size reported as hydrodynamic diameter was obtained by cumulative analysis. To determine the zeta potential, samples were resuspended $(50 \mu \mathrm{l})$ into $0.1 \mathrm{mM} \mathrm{NaCl}(950 \mu \mathrm{l})$ using folded capillary cells for zeta analysis. The Smoluchowski approximation was used to support the calculation of the zeta potential from the electrophoretic mobility. Zeta potential measurements were run in triplicate. Only data that met the quality criteria according with the software program (DTS 5.0) were included in the study.

\subsection{Agarose gel electrophoresis studies.}

Naked DNA and nioplexes based on different helper lipids and without helper lipid at 30/1 cationic lipid/DNA (w/w) mass ratio (containing $200 \mathrm{ng}$ of the plasmid) were subjected to agarose gel electrophoresis. The agarose gel (0.8\%) (Sigma-Aldrich, Madrid, Spain) was 
immersed in a Tris-acetate-EDTA buffer and exposed for $30 \mathrm{~min}$ to $120 \mathrm{~V}^{\text {V GelRed }}{ }^{\mathrm{TM}}$ (Biotium, Hayward, California, USA) was used to stain the DNA bands and images were observed with a ChemiDoc $^{\mathrm{TM}}$ MP Imaging System and analyzed by Image Lab ${ }^{\mathrm{TM}}$ Software (BioRad, USA). To evaluate the release of DNA from the formulation, $12 \mu \mathrm{l}$ of a $7 \%$ sodium dodecyl sulfate (SDS) (Sigma- Aldrich, Madrid, Spain) solution was added to the samples. The DNA protection capacity of the vectors against enzymatic digestion was analyzed by adding the DNase I enzyme (Sigma-Aldrich, Madrid, Spain) to the formulations (final concentration of $1 \mathrm{U}$ DNase I per $2.5 \mu \mathrm{g}$ DNA). Afterward, the mixtures were incubated at $37 \stackrel{\circ}{\circ} \mathrm{C}$ for $30 \mathrm{~min}$. Finally, $12 \mu \mathrm{l}$ of a $7 \%$ SDS solution was added to analyze the released DNA. Each sample contained $4 \mu \mathrm{l}$ of loading buffer (BioRad, USA). Untreated DNA was used as a control to compare the integrity of the DNA in each sample.

\subsection{Cell culture for transfection and cell uptake studies.}

ARPE-19 cells (ATCC) were grown in D-MEM/F-12 containing $10 \%$ bovine serum and supplemented with $5 \%$ penicillin-streptomycin (Gibco1 San Diego, California, USA) and incubated at $37 \stackrel{\circ}{\mathrm{C}}$ and $5 \% \mathrm{CO}_{2}$ atmosphere. Cells were split every 3-4 days to maintain monolayer coverage. For transfection and cell uptake assays, the cells were seeded in 24 well plates at initial density of $10 \times 10^{4}$ cells per well, with $300 \mu$ l of D-MEM/F-12 containing $10 \%$ bovine serum and incubated at $37 \stackrel{\circ}{\circ} \mathrm{C}$ and $5 \% \mathrm{CO}_{2}$ atmosphere. Cells were adhered overnight to achieve $70-90 \%$ of confluence at the time of transfection and cell uptake assays. For transfection assay, the regular growth medium was removed from ARPE-19 cells, and the cells were exposed to nioplexes based on the different helper lipids and without helper lipid (containing $1.25 \mu \mathrm{g}$ of the plasmid for each well) resuspended in Opti-MEM ${ }^{\mathrm{TM}}$ transfection medium (Gibco ${ }^{\mathrm{TM}}$, San Diego, California, USA). Each formulation was used in triplicate. After 4 $h$ of incubation, the nioplexes were removed. Then, cells were washed twice with PBS (SigmaAldrich, Madrid, Spain) and $300 \mu \mathrm{l}$ of regular growth medium was added to the cells (Ojeda et al., 2015a). Cells were allowed to grow for $72 \mathrm{~h}$ until flow cytometry analysis. For cell uptake assay, the regular growth medium was removed from ARPE-19 cells, and the cells were exposed to nioplexes prepared with different helper lipids and without helper lipid (containing $1.25 \mu \mathrm{g}$ of Cy3-labeled plasmid for each well) resuspended in Opti-MEM ${ }^{\mathrm{TM}}$ transfection medium. After $1 \mathrm{~h}$ of incubation, the nioplexes were removed. Then, cells were washed twice with PBS and detached for flow cytometry analysis. Each formulation was used in triplicate.

\subsection{Transfection efficiency and cell viability assays.}

FACSCalibur system flow cytometer (Becton Dickinson Bioscience, San Jose, USA) was used to conduct flow cytometry analysis to quantify the \% of EGFP and the mean fluorescence intensity (MFI) of positive cells. Cells were washed twice with PBS (Sigma-Aldrich, Madrid, Spain) and detached from the microplate with $200 \mu \mathrm{l}$ of trypsin/EDTA (Gibco ${ }^{\mathrm{TM}}$ San Diego, California, US). Once the cells were detached, $400 \mu$ l of normal growth medium was added and directly introduced into the flow cytometer tubes. Transfection efficiency was expressed as the percentage of EGFP positive cells at $525 \mathrm{~nm}$ (FL1) after excluding dead cells stained with $5 \mathrm{ml}$ 
of diluted (1/20) Sytox ${ }^{\text {TM }}$ Blue reagent (Invitrogen, Carlsbad, CA, USA). Control samples (nontransfected cells) were displayed on a forward scatter (FCS) versus side scatter (SSC) dot plot to establish a collection gate and exclude cell debris. The fluorescence corresponding to dead cells was measured at $444 \mathrm{~nm}$ (FL6). Mean fluorescence intensity (MFI) data were obtained from live positive cells (FL1). Control samples containing Lipofectamine ${ }^{\mathrm{TM}} 2000$ (Invitrogen, Carlsbad, CA, USA), transfected cells without Sytox ${ }^{\top M}$ blue, and non-transfected cells with Sytox ${ }^{\top \mathrm{M}}$ blue were used to established the cytometer settings and channel compensations. For each sample, 10000 events were collected. Each formulation was used in triplicate. FlowJo software (Becton Dickinson, Mountain View, CA, USA) was used to analyze the data.

\subsection{Cell uptake analysis.}

FACSCalibur system flow cytometer was used to conduct flow cytometry analysis to quantify the percentage of cells that contained Cy3-labeled plasmid DNA. Cells were washed twice with PBS and detached from the microplate with $200 \mathrm{ml}$ of trypsin/ EDTA. Once the cells were detached, $400 \mathrm{ml}$ of normal growth medium were added and directly introduced into the flow cytometer. Cell uptake was expressed as the percentage of Cy3- labeled plasmid positive cells at $585 \mathrm{~nm}$ (FL2) after excluding dead cells as previously described. Mean fluorescence intensity (MFI) data were obtained from live positive cells (FL2). Control samples (nontransfected cells) were displayed on a forward scatter (FSC) versus side scatter (SSC) dot plot to establish a collection gate and exclude cells debris. For each sample, 10000 events were collected. Each formulation was used in triplicate. Data was analyzed by FlowJo software (Becton Dickinson, Mountain View, CA, USA).

\subsection{Internalization mechanism assays.}

To identify the endocytic vesicles involved in nioplexes internalization, we performed colocalization assays in ARPE- 19 cells. The cells were seeded in 12 well plates containing coverslips at an initial density of $20 \times 10^{4}$ cells per well. A volume of $600 \mu \mathrm{l}$ of D-MEM/F-12 containing $10 \%$ bovine serum was added to each well and incubated at $37 \stackrel{\circ}{\circ} \mathrm{C}$ and $5 \% \mathrm{CO}_{2}$ atmosphere. Cells were adhered overnight to achieve $70-90 \%$ of confluence at the time of internalization assay. Then, the regular growth medium was removed to expose the cells to nioplexes based on the different helper lipids and without helper lipid (containing $0.625 \mu \mathrm{g}$ of the Cy3 plasmid for each well) and fluorescent endocytic markers. Briefly, the markers were used as follows (Cardarelli et al., 2012): $60 \mu \mathrm{l}(10 \mathrm{mg} / \mathrm{ml}$ ) of Dextran Alexa Fluor 488, a fluidphase uptake marker, incubated for $60 \mathrm{~min}$ to label macropinosomes; $96 \mu \mathrm{l}(0.5 \mathrm{mM})$ of Lysotracker ${ }^{\mathrm{TM}}$ Green incubated for 60 min to label lysosomes; $5 \mu \mathrm{l}(1 \mathrm{mg} / \mathrm{ml})$ of Cholera toxin B Alexa Fluor 488 incubated for $60 \mathrm{~min}$ to label CVME; and $5 \mu \mathrm{l}(5 \mathrm{mg} / \mathrm{ml})$ of Transferrin Alexa Fluor 488 incubated for $60 \mathrm{~min}$ to label CME. All markers were purchased from Life Technologies, Eugene, OR, USA. Once the markers and the nioplexes were added, Opti-MEM ${ }^{\mathrm{TM}}$ transfection medium was incorporated to obtain $600 \mathrm{ml}$ final volume in each well. Cells were incubated for $1 \mathrm{~h}$ at $37 \stackrel{\circ}{\mathrm{C}}$ and $5 \% \mathrm{CO}_{2}$ atmosphere. Next, the medium containing nioplexes was removed, and cells were washed twice with PBS. Then, the cells were fixed with $4 \%$ 
formaldehyde and mounted for their posterior examination by confocal microscopy. ImageJ software (NIH Image; http://rsbweb.nih.gov/ij/) was used to measure colocalization through Mander's overlap coefficient between red-labeled nio-plexes and green stained endocytic pathways. ( $\mathrm{M}$ = colocalized red/ total red). Colocalization measurements were evaluated in $\mathbf{3 0}$ cells for each entry pathway.

\subsection{Confocal laser scanning microscopy experiments (CLSM).}

CLSM experiments were performed to evaluate qualitatively and quantitatively the colocalization between the different nioplexes formulations and the Cy3 stained DNA. Such assays were carried out with the Olympus Fluoview 1000 confocal microscope (Olympus, Tokyo, Japan) interfaced with a $405 \mathrm{~nm}$ diode laser, a $488 \mathrm{~nm}$ Argon laser, and $543 \mathrm{~nm} \mathrm{HeNe}$ laser to excite the fluorescently labeled pDNA. Mounted slides were viewed with a $60 \times 1.25$ numerical aperture (NA) water immersion objective. The following collection ranges were adopted: 500-540 nm (EGFP, Transferrin Alexa Fluor 488, Cholera toxin B Alexa Fluor 488 and Dextran Alexa Fluor 488), 555-655 nm (Cy3), and 460-530 (Lysotracker ${ }^{\mathrm{TM}}$ Green). Images were collected in a sequential mode to eliminate emission crosstalk between the dyes.

\section{Results and discussion}

\subsection{Synthesis of the ionizable cationic amino lipid.}

The evaluation of acid dissociation constants (pKa) in cationic lipids has become a useful parameter to design more efficient $\mathrm{pH}$-responsive lipid-based drug delivery systems (Jayaraman et al., 2012). This mechanism normally benefits the release of the delivery system cargo in the presence of acid $\mathrm{pH}$ caused by late endosomes ( $\mathrm{pH} 6.5)$ or lysosomes ( $\mathrm{pH} 4.5)$. This property has allowed the design and synthesis of ionizable cationic lipid combinatorial libraries and formulations, which have shown a significant role on mediating cellular internalization and thereby causing the expected gene silencing in vitro and in vivo (Jayaraman et al., 2012). In our previous work (Ojeda et al., 2015b), we found a tight correlation between acid dissociation constant (pKa) value and activity of cationic lipid-based combinatorial libraries, where an optimization was determined by several variables like lipid-chain unsaturation, linker chemistry and polar head nature. Interestingly, an optimum pKa $(<7.0)$ for transfection was established as an ideal value to design new liposomal drug delivery systems (Semple et al., 2010). Specifically, this optimal relationship between pKa (6.72) and silencing activity was successfully found in the serinol isomer of the presented compound with the dimethylaminoethyl pendant group (Ojeda et al., 2015b). These findings made us to consider the possibility of introducing this potential modification to the presented cationic lipid: 1-(2dimethylaminoethyl)-3-[2,3-di(tetradecyloxy)propyl] urea and continue with a deeper characterization. Moreover, this cationic lipid has already shown interesting transfection efficiencies in vivo in rats (Ojeda et al., 2015a).

In light of the promising transfection results in vitro and in vivo recently obtained by our research group with optimized formulations based on the amino lipid 2,3- 
di(tetradecyloxy)propan- 1-amine (Puras et al., 2014) (1, Fig. SI 1), we decided to chemically modify our glycerol-based cationic lipid lead compound with other potential polar head groups like this dimethylaminoethyl moiety used for gene delivery purposes.

The introduction of the dimethylamino derivative was accomplished by activating the amino lipid 1 with p-nitrophenyl- chloroformate according to well-stablished protocols (Ojeda et al., 2015b). Final nucleophilic reaction introduced the anticipated cationic head group and generated the expected urea derivative in moderate yields (65\%) (Fig. SI 1). Having in hand the expected cationic lipid compound, we focused on evaluating the most appropriate niosomal formulation by adding squalene, cholesterol and squalane as helper lipids and its efficiency in gene transfection experiments.

\subsection{Size polydispersity index and zeta potential measurements of niosomes and nioplexes.}

Combining size and zeta potential measurements is one of the most experimental strategies used to characterize the formation of vector/DNA complexes. Therefore, we investigated such param-eters along with the polydispersity index (Fig. 2). Size characterization (Fig. 2A, white bars) indicated that niosomes based on squalene and cholesterol showed similar size (147 and $176 \mathrm{~nm}$, respectively). Niosomes based on squalane showed the biggest size $(259 \mathrm{~nm})$ and niosomes prepared without helper lipid the smallest size $(61 \mathrm{~nm})$. As a homogeneity parameter for the niosome formulations, we determined the polydispersity index (PDI) (Fig. 2B). All niosome formulations showed appropriate size homogeneity with PDI values lower than 0.372 . Zeta potential values of niosomes prepared with different helper lipids are shown in Fig. 2A (dot lines). The results showed that the zeta potential value obtained for niosomes prepared with squalene and squalane were similar (around $+50 \mathrm{mV}$ ). For niosomes prepared with cholesterol, the zeta potential value was around $+40 \mathrm{mV}$. Finally, the lowest values were observed with niosomes prepared without helper lipid $(+32 \mathrm{mV})$. The cationic lipid/DNA ratio $\mathrm{w} / \mathrm{w}$ employed to form the nioplexes was the result from a previous characterization study (data not shown), where the ratio 30/1 was found the most appropriated. The size of the nioplexes (Fig. 2A gray bars) showed the following results: Nioplexes based on squalene (150 nm), cholesterol (180 nm), squalane $(254 \mathrm{~nm})$, and without helper lipid $(79 \mathrm{~nm})$. These data indicate that the addition of DNA to niosomes to form nioplexes does not modify remarkably the original size of the niosomes, probably due to the high DNA condensation capacity of all formulations, with the exception of the niosome formulation prepared without helper lipid. All the formulations showed acceptable PDI values lower than 0.369 (Fig. 2B). The addition of the negatively charged DNA to niosomes decreased the zeta potential of all nioplexes formulations (Fig. 2A dash lines): Nioplexes based on squalene (from +50 to $+40 \mathrm{mV}$ ), nioplexes based on cholesterol (from +38 to $+35 \mathrm{mV}$ ), nioplexes based on squalane (from +51 to $+46 \mathrm{mV}$ ) and, especially in nioplexes prepared without helper lipid (from +32 to $+14 \mathrm{mV}$ ). This reduction on the superficial charge could be explained in part by the partial neutralization of the cationic charge carried by niosomes by the negatively charged DNA molecules. In any case, all nioplexes molecules were found to be positively charged (Fig. 2A dash lines), which suggest that at this cationic lipid/DNA mass ratio $(30 / 1, w / w)$ free DNA is not present on the formulation and all the DNA is bound to the 
niosome. Collectively, our data shows that size and zeta-potential results are compatible with DNA binding to niosome surfaces (Caracciolo and Caminiti, 2004). The addition of DNA to the niosomes did not remarkably modify the particle sizes, which are adequate for cellular uptake and internalization purposes (Caracciolo et al., 2009; Gillard et al., 2014; Gratton et al., 2008; Lim and Gleeson, 2011; Rejman et al., 2004; Xiang et al., 2012). Regarding zeta potential, it must be highlighted that all formulations showed positive zeta-potential values, which enhances not only the formation of stable suspensions due to the electrostatic repulsion between the positively charged particles (Caracciolo and Amenitsch, 2012) but also the interaction with negatively charged cell surfaces and the subsequences cell uptake processes (Rezvani Amin et al., 2013). In any case, we have to consider as well that both particle size and zeta potential values were measured as previously reported (Ojeda et al., 2015a; Puras et al., 2014 ) in $0.1 \mathrm{mM} \mathrm{NaCl}$ medium to avoid saturation of the electrodes in the cuvettes. Therefore, although obtained values have significant importance for comparative purposes among the different formulations, absolute values could differ from those obtained in other fluids, such as transfection medium or other biological fluids, due to differences in the osmolarity or $\mathrm{pH}$ values to name a few.

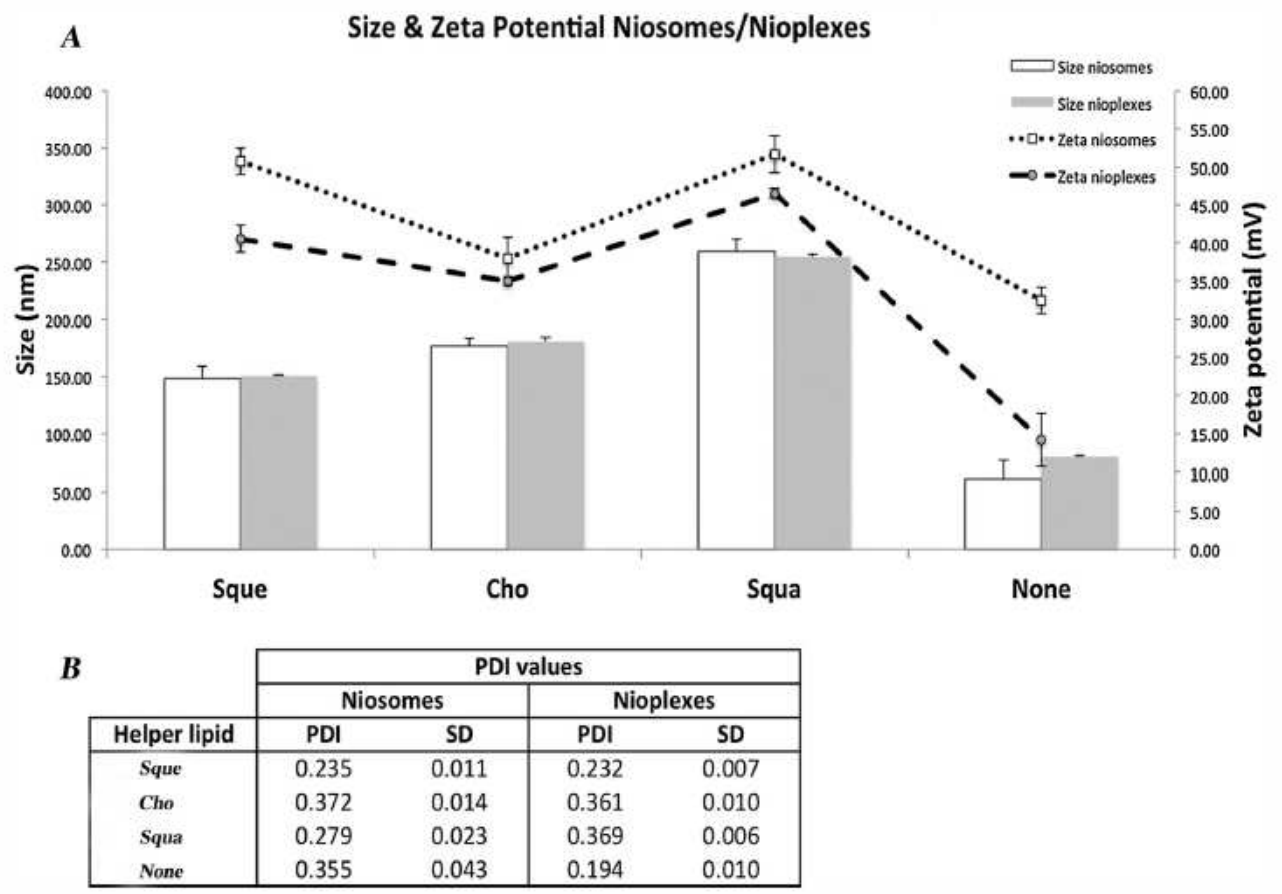

Fig. 2. Physico-chemical characterization of niosomes and nioplexes (niosome/DNA w/w ratio 30/1) prepared with different helper lipids: squelene (Sque), cholesterol (Cho), squalane (Squa) and without helper lipid (None). A) Size (white bars) and zeta potential (dot line) of niosomes. Size (gray bars) and zeta potential (dash lines) of nioplexes. B) PDI values of niosomes and nioplexes. Each value represents the mean \pm standard deviation of three measurements.

\subsection{Agarose gel electrophoresis studies.}


We investigated the electrostatic interactions of niosomes and DNA, the release of the DNA by SDS addition to the formulations and the capacity of niosomes to protect DNA against enzymatic digestion (Fig. 3) (Ochoa et al., 2014; Ojeda et al., 2015b). The supercoiled (SC) bands in the gel show the most bioactive DNA form, and open circular (OC) bands represent the structural change of DNA of a less active form (Stellwagen and Stellwagen, 2009).

Nioplexes based on squalene showed deficient DNA condensation (lane 4), as a faint $\mathrm{SC}$ band was shown in the agarose gel. An easy release of DNA from the niosomes was detected (lane 5) upon the addition of the SDS surfactant agent, which could be the consequence of poor condensation. Due to the SC band observed in lane 6 (treatment with SDS plus DNase I), we can conclude that nioplexes based on squalene were able to protect the DNA from enzymatic digestion.

In the case of nioplexes based on cholesterol, we observed that they were able to condensate the DNA (lane 7). Moreover, we observed partial release of DNA upon the addition of SDS (lane 8) as all DNA content was not observed at the bottom of the well. Reasonable DNA protection was also observed as indicated by the detected SC band (lane 9).

The analysis of nioplexes based on squalane, indicated high DNA condensation (lane 10), probably, due to their observed high zeta potential values (+46 mV, Fig. $2 \mathrm{~A}$ dash lines). Moreover, these nioplexes were able to release the DNA efficiently upon the addition of SDS (lane 11). Concerning about DNA protection against enzymatic digestion, these nioplexes showed that DNA was properly protected (lanes 12).

Finally, nioplexes prepared without helper lipid, despite their low zeta potential values (+14 mV, Fig. 2A dash lines), showed high DNA condensation capacity (lane 13). Thus, it seemed that the absence of helper lipids in the formulations could increase the condensation of DNA at low zeta potential values. Additionally, there was a satisfactory release and protection of DNA from enzymatic digestion, as SC bands were observed in lanes 14 and 15, respectively.

In brief, these studies showed that nioplexes prepared without helper lipid showed the highest level of DNA condensation whereas nioplexes based on squalene showed the lowest one. About DNA release, nioplexes based on squalene, squalane and without containing helper lipid showed an excellent capability to release the DNA. It is worth mentioning that both condensation and release of DNA are important parameters that need to be evaluated for the success of nioplexes as gene delivery vectors, where a delicate balance between these two factors for proper DNA delivery is required (Puras et al., 2014).

DNA protection is another factor to take into account due to the presence of enzymes in the cytosol since they can easily degrade the DNA and thus hamper the desired DNA delivery process (Midoux and Monsigny, 1999). This DNA protection was observed for all niosome formulations, which were able to protect the DNA against enzymatic digestion 


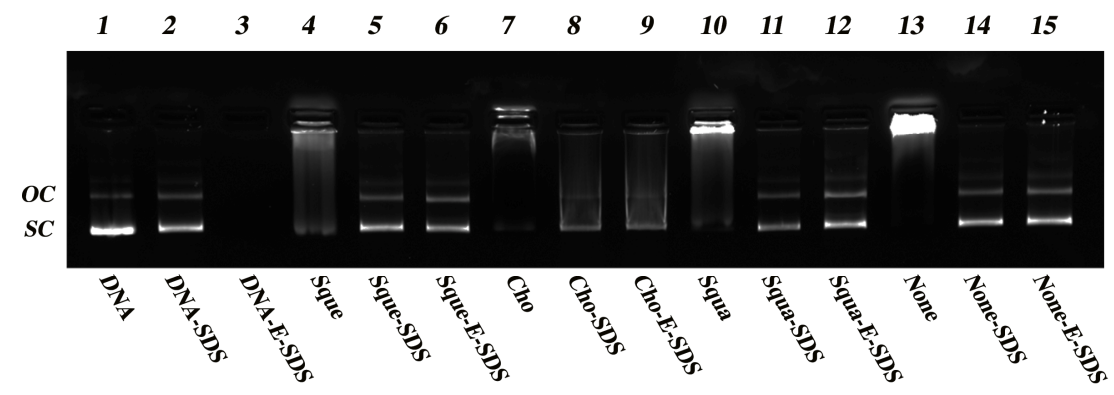

Fig. 3. Binding, SDS-induced release and protection of DNA prepared with different helper lipids: squelene (Sque), cholesterol (Cho), squalane (Squa), without helper lipid (None). OC: open circular form, SC: supercoiled form. Lanes 1-3 correspond to free DNA; lanes 4-6, nioplexes based on squalene; lanes 7-9, nioplexes prepared with cholesterol; lanes 10-12, nioplexes based on squalane; lanes 13-15, nioplexes prepared without helper lipid. Naked DNA and nioplexes were treated with SDS (lanes 2,5,8,11 and 14) and DNase I + SDS (lanes $3,6,9,12$ and 15).
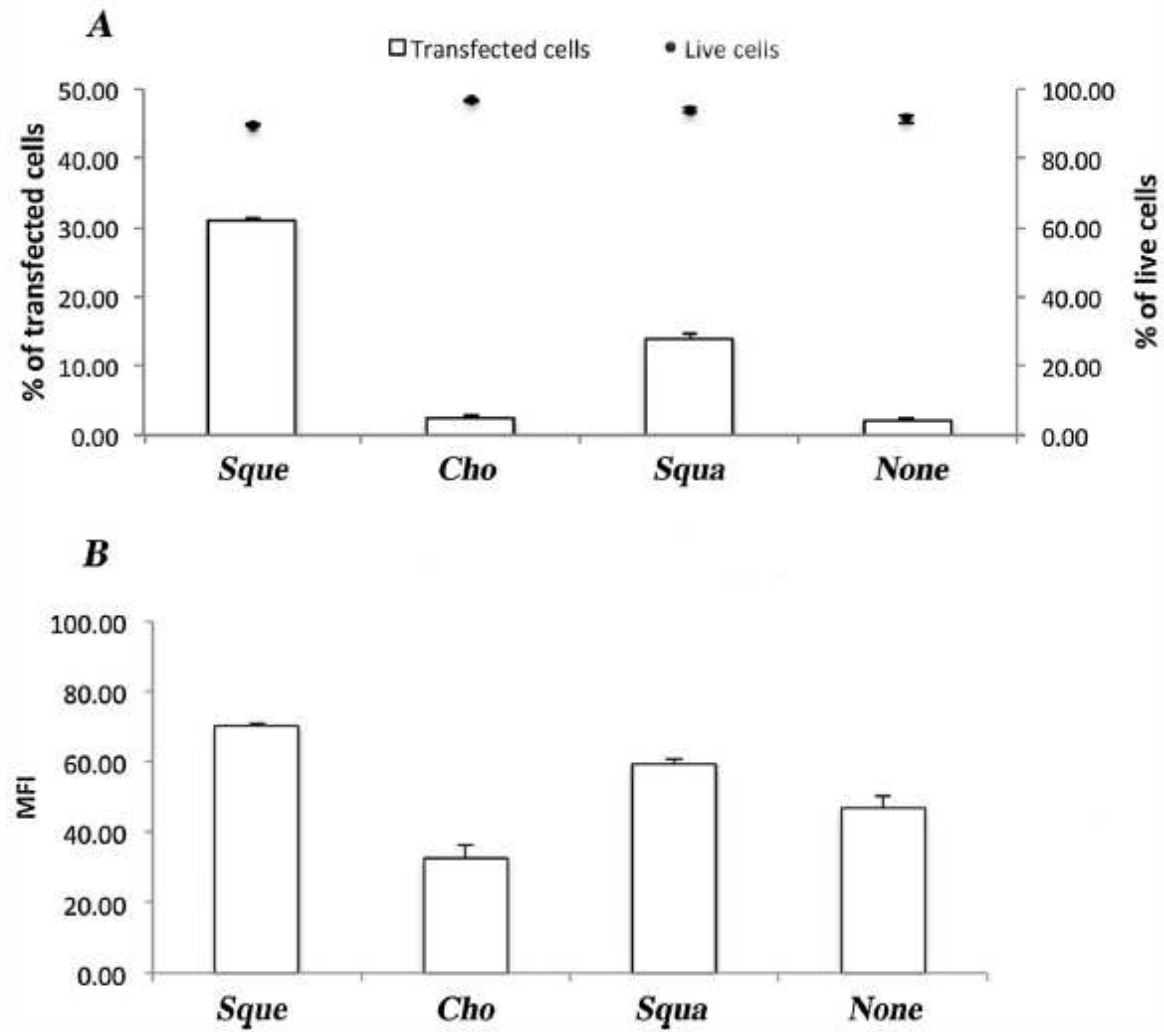

Fig. 4. Transfection efficiencies at $72 \mathrm{~h}$ post-addition of nioplexes prepared with different helper lipids: squalene (Sque), cholesterol (Cho), squalane (Squa) and without helper lipid (None) in ARPE-19 cells. A) Percentage of transfection (bars) and viability (dots) of nioplexes. B) Mean fluorescence intensity (MFI) of cells with nioplexes prepared with different helpers. Each value represents the mean \pm standard deviation of three measurements. 


\subsection{In vitro transfection and cell uptake experiments.}

Percentages of transfected cells, cell viability, and cellular uptake studies were carried out in ARPE-19 cells as a retinal cell model for in vivo applications (Ochoa et al., 2014; Puras et al., 2013). These pigmented epithelial cells of the retina play a major role in retinal diseases associated with senescence and dystrophies of the photoreceptors. Mutations in genes of these cells can lead to photoreceptors death with dramatic consequences (Bejjani et al., 2005). Therefore, the knowledge of the physicochemical and biological parameters that affect to the transfection efficiency on these particular cells merits special attention in order to design more efficient non-viral vectors for retinal gene delivery applications (Ojeda et al., 2015a).

Gene expression in the cell nucleus is an indication that the DNA has been properly delivered by the vector. However, this process can involve, sometimes, few numbers of cells, which can be inadequate for gene therapy purposes. Therefore, our aim is to transfect as many cells as possible without compromising the cellular viability. The highest percentages values of transfected cells were observed for nioplexes prepared with squalene $(31 \%)$, followed by nioplexes prepared with squalane (14\%) (Fig. 4A bars). The lowest transfection values were obtained when nioplexes were prepared with cholesterol (2\%) and without helper lipid (2\%). For additional transfection data see histograms in supporting infor- mation (Fig. SI 2). Therefore, and as reported in the literature, transfection efficiency strongly depends on the chemical compo-sition of the helper lipids (Chung et al., 2001; Kim et al., 2003). The above results could be explained, in part, by the different endosomal scape ability of the helper lipids (Pozzi et al., 2012). This capability has been attributed to the structural transition that helper lipids offer to the delivery system. Such structural transition usually goes from lamellar to inverse hexagonal due to the acid environment of the endosomes, where the transition induces the release of the DNA and its subsequent delivery into the cytoplasm (Allain et al., 2012; Zuhorn et al., 2005). Additionally, analyzed MFI data (Fig. 4B) showed that nioplexes prepared with squalene showed the higher MFI, around 70. On the other hand, despite of the low percentage of transfected cells observed with nioplexes prepared with the other three formulations (Fig. 4A bars), we found interesting MFI data that suggest that the few number of transfected cells were able to produce significant amounts of EGFP (Fig. 4B, Cho, Squa and None). The capacity of nioplexes based on squalene to transfect more efficiently ARPE-19 cells might also rely on the fact that squalene is usually synthesized in the cells, which may indicate that cells could easily recognize it and subsequently increase its cell permeability (Koivisto and Miettinen 1988; Liu et al., 1976; Stewart, 1992). Therefore, we hypothesize that nioplexes prepared with squalene could take advantages of the properties of this helper lipid to obtained high transfection efficiencies. In any case, it could be interesting to perform further in vivo experiments since transfection efficiency in vitro conditions may be strongly different than the efficiency observed in real biological fluids, mainly due to unspecific interactions between gene delivery vectors and biomolecules, such as serum proteins, that clearly affect to the stability and uptake of the formulations (Puras et al., 2013). In any case, we have recently reported that niosomes based on squalene helper lipid transfected efficiently both the rat retina after intravitreal and subretinal administrations and the rat brain (Ojeda et al., 2015a; Puras et al., 2014). 
Additionally, to develop efficient drug delivery systems, it is important to consider the possible toxic effect of these vectors upon the targeted cells since cell viability generally depends on the vector composition (Martin et al., 2005; Ojeda et al., 2015b). Therefore, the effect of our niosome formulations on ARPE-19 cells was studied. Cell viability results (Fig. 4A dots) showed high viabilities with all the nioplexes formulations (around 90\%), which indicated high cell tolerability with all the helper lipids used in each formulation. Cell uptake analysis is another primary assay that helps to understand part of the transfection process and shows the capacity of the nioplexes to be internalized when they are in contact with the cells.

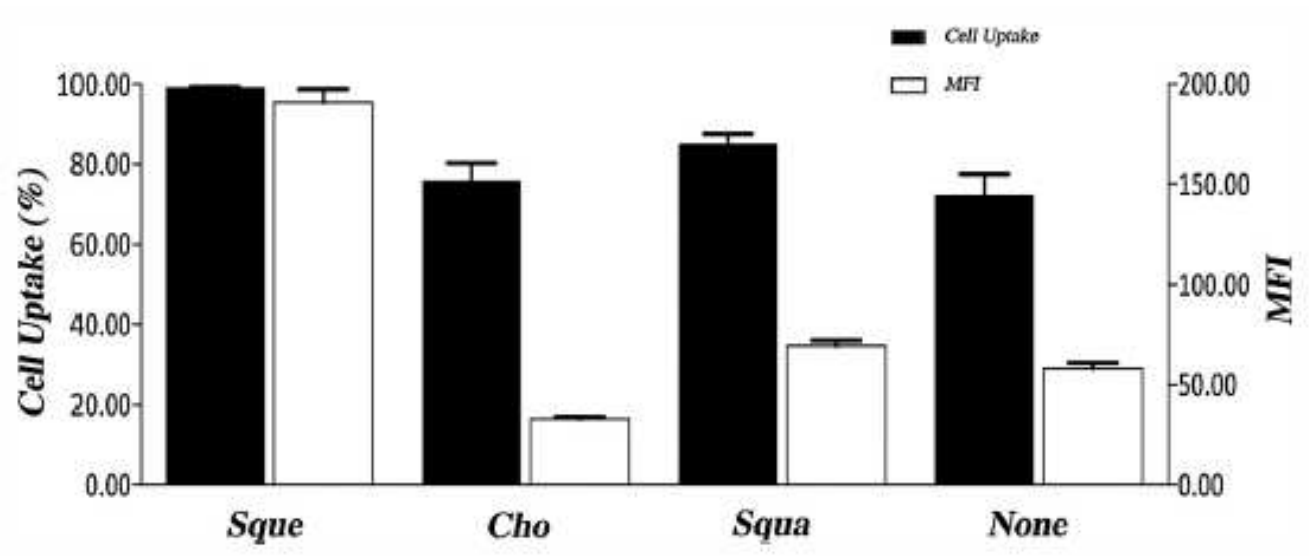

Fig. 5. Cellular uptake at $1 \mathrm{~h}$ after the addition of nioplexes prepared with different helper lipids: squalene (Sque), cholesterol (Cho), squalane (Squa) and without helper lipid (None) in ARPE-19 cells. Uptake percentage of cells upon the addition of nioplexes (Black bars). Mean fluorescence intensity (MFI) of cells with nioplexes prepared with different helpers (White bars). Each value represents the mean \pm standard deviation of three measurements.

Cell uptake percentages results (Fig. 5 black bars) showed that nioplexes based on squalene were practically internalized by all the cells (99\%), and nioplexes based cholesterol, squalane and without helper also showed worthy cell uptake levels $(75 \%, 85 \%$ and $72 \%$, respectively). For additional uptake data see histograms in supporting information (Fig. SI 3). To show a better insight into the cell uptake process, we analyzed as well the mean MFI of the cells (Fig. 5 white bars). Our results showed that nioplexes based on squalene helper lipid showed higher MFI (around 200) compared to the rest of formulations (nioplexes based on cholesterol 32, squalane 69 and without helper lipid 58). Thus, our cellular uptake studies indicated that nioplexes based on squalene were internalized in most of the analyzed cell population, and the amount of nioplexes internalized in each cell was superior compared to the rest of formulations. Regarding this point, we could assume that the higher MFI value of niosomes based on squalene observed in Fig. 5 (white bars) could be due to the higher particle size of this formulation rather than to the differences on number of particles internalized. However, data reported on Fig. 2A, clearly discard this assumption. Moreover, we could discard the hypothesis that only Cy3-labelled DNA without the niosomes could enter the cell since data reported on Fig. $2 \mathrm{~A}$ shows that at 30/1 cationic lipid/DNA mass ratio all the DNA is bound to the niosome. 

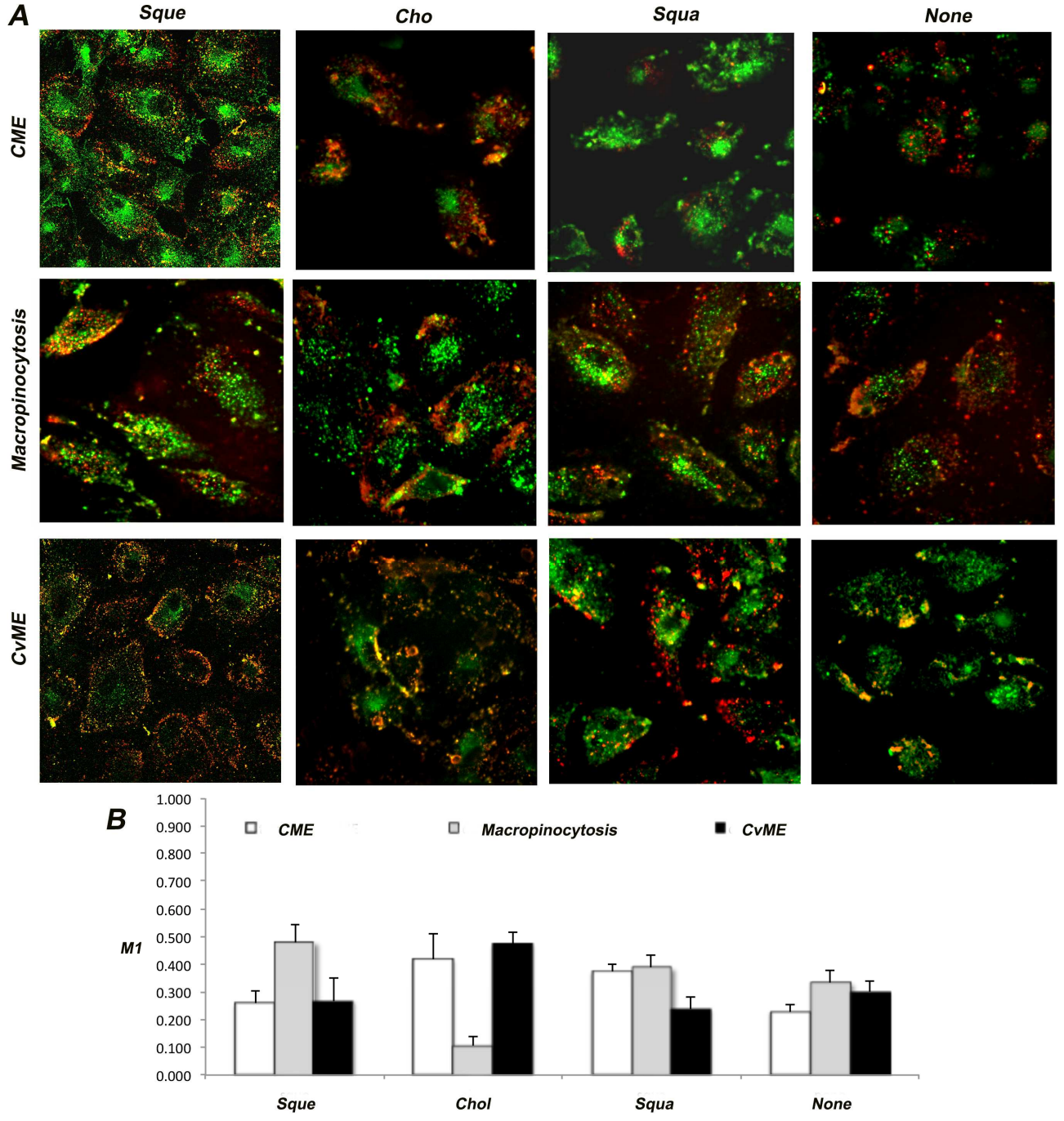

Fig. 6. Cell trafficking results in ARPE-19 cells co-incubated with nioplexes prepared with squalene (Sque), cholesterol (Cho), squalane (Squa) and without helper lipid (None). A) Confocal microscopy merged images showing the cells co-incubated with nioplexes. Each image displayed in the optical section was representative of the cell population. Green coloring shows cells stained with Transferrin Alexa Fluor 488 for CME, Dextran Alexa Fluor 488 for macropinocytosis and Cholera toxin B Alexa Fluor 488 for CvME. Red coloring shows the DNA stained with Cy3. B) Colocalization values were given as the fraction of cell-associated nanoparticles colocalizing with fluorescently labeled endocytic structures. M1 is equal to Mander's overlap coefficient between the red signal from the stained DNA and the green fluorescence of the stained entry pathways. Each value represents the mean \pm standard deviation of three measurements. (For interpretation of the references to color in this figure legend and text, the reader is referred to the web version of this article.)

Additionally, fluorescent confocal microscopy images showed that immediately after administration ( $t<5 \mathrm{~min}$ ), DNA appeared to be confined within punctate spot-like structure, 
while no evidence of diffuse fluorescence was found (see Supporting information Figure SI 4). This finding unambiguously suggested that, at the early stages of internalization, DNA was encapsulated within niosomes vesicles. Additionally, squalene has shown to increase the phagocytic activity of cells due to its capacity to potentiate the immune response in a nontoxic manner (Allison and Byars, 1986; Seubert et al., 2008). Furthermore, some authors have reported that squalene increases oxygenation in the cells, which results in a more efficient metabolic process (Reddy and Couvreur, 2009). Therefore, a more efficient and easy uptake of DNA could be performed when squalene is used as helper lipid in our niosome formulations.

Among many other parameters, cell uptake could also depends on physico-chemical parameters of the niosomes such as the particle size, where bigger size particles $(>500 \mathrm{~nm}$ ) are difficult and slowly taken, contrary to smaller particles $(40 \mathrm{~nm})$, which are easily and rapidly captured (dos Santos et al., 2011). However, our results indicated that the lowest cell uptake value was obtained from the smallest particles $(79 \mathrm{~nm}$, nioplexes prepared without helper lipid), and the most efficient cellular uptake was obtained with bigger nioplexes (around 150 $\mathrm{nm}$, nioplexes prepared with squalene and cholesterol). These data suggest that not only size is determinant in the cellular uptake but the helper lipid composition as well.
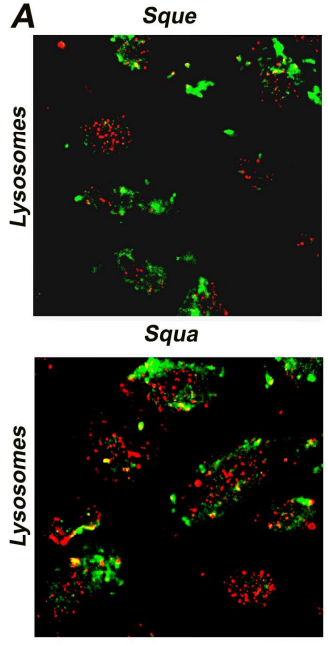
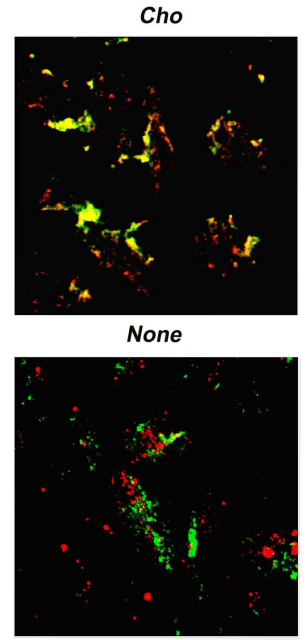
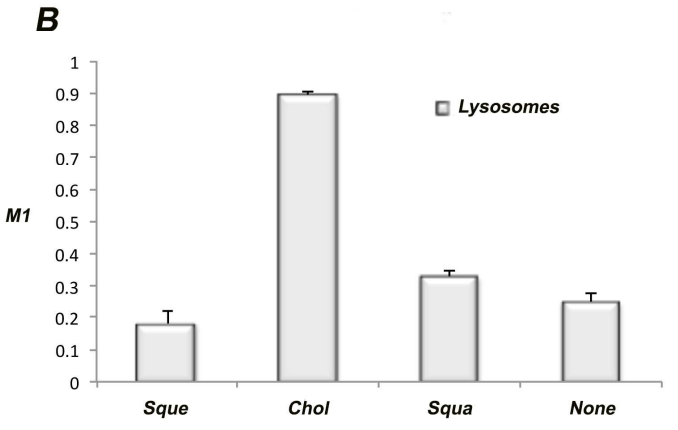

Fig. 7. Lysosome colocalization results in ARPE-19 cells co-incubated with nioplexes prepared with squalene (Sque), cholesterol (Cho), squalane (Squa) and without helper lipid (None). Niosomes were complex with DNA stained with Cy3. A) Confocal microscopy merged images showing cells co-incubated with nioplexes prepared with different helper lipids. Each image displayed in the optical section was representative of the cell population. Green and red coloring show cells stained with Lysotracker1 Green and the DNA, respectively. B) The colocalization values were given as the fraction of cell-associated nanoparticles colocalizing with fluorescently labeled lysosomes, where M1 is equal to Mander's overlap coefficient between the red signal from the stained DNA and the green fluorescence of the stained lysosomes. Each value represents the mean \pm standard deviation of three measurements. (For interpretation of the references to color in this figure legend, the reader is referred to the web version of this article.) 


\subsection{Intracellular trafficking.}

To develop more efficient non-viral vectors it is necessary to understand their intracellular trafficking mechanism and how their physical-chemical properties might trigger different internalization pathways. Thus, in the following experiments, we have investigated the intracellular traffic of nioplexes formulations and how this process influences the transfection efficiency. Fluorescence confocal microscopy allowed visualizing the intra-cellular trafficking of fluorescently labeled nioplexes (red) in ARPE- 19 cells in the presence of various endocytic markers (green) (Fig. 6A). Since there was the possibility of finding dextran-positive endosomes internalized by clathrin-mediated pathway (roughly 100-200 nm in size), the incubation time applied (60 $\mathrm{min}$ ) was chosen to minimize accumulation of dextran in endosomes internalized by mechanisms other than macropinocytosis. The degree of colocalization was calculated by Mander's overlap coefficient (Fig. 6B) to perform a quantitative analysis of confocal images. Colocalization analysis (Fig. 6B) showed that squalene- containing nioplexes mostly used macropinocytosis $(M=0.480)$ followed by CVME $(M=0.266)$ and $C M E(M=0.258)$. On the other side, cholesterol-containing nioplexes were mainly located in CME $(M=0.420)$ and CVME $(M=0.475)$, while they were poorly processed throughout macropinocytosis $(M=0.106)$. Nioplexes prepared with squalane, were internalized via macropinocytosis $(M=0.388)$ and $C M E(M=0.376)$ and to a less extent via CvME ( $M=0.237)$. Lastly, in the absence of helper lipid nioplexes were also equally internalized via CME ( $M=0.227)$, macropinocytosis $(M=0.336)$ and CVME ( $M=0.300)$. Our results indicated that each nioplexe formulation could use all the entry pathways simultaneously. Notably, transfection efficiencies did correlate to the internalization pathway: the larger the involvement of macro-pinocytosis, the higher the transfection efficiency. To account for differences in transfection efficiency the ultimate intracellular fate of nioplexes was investigated (Fig. 7A). To this end, we used Lysosensor, which accumulates in acidic cell organelles, and is primarily a lysosome marker. Colocalization of fluorescently tagged nioplexes and Lysosensor reveals that highly efficient squalene-containing systems could efficiently escape from endo-somal compartments $(M=0.180)$, while poorly efficient cholester- olcontaining nioplexes were largely degraded in the lysosomes $(M=0.895)$. Nioplexes prepared either with squalane and with no helper lipid exhibited intermediate levels of lysosomal accumula-tion ( $\mathrm{M}=0.330$ and 0.247 , respectively).

The precise correlation between the mechanism of cell uptake, final intracellular fate and transfection efficiency of nioplexes has been poorly investigated so far. It has been suggested that $\mathrm{CME}$ and macropinocytosis are correlated to the lysosomal fusion, which could partial or entirely degrade the content of their vesicles (Luzio et al., 2009; Xiang et al., 2012). Moreover, it has been described that there is a recycling process of the vesicles when macropinocytosis is involved (i.e. macropinosome is sent back to the cell exterior) (Lim and Gleeson, 2011). It also should be pointed out that CvME is mainly considered a non-digestive pathway. However, some findings suggest that this route is also involved in the lysosomal fusion (Kiss and Botos, 2009).

In this regard, our results provided new views and insights into the relationship between intracellular trafficking and transfection efficiency of nioplexes. Squalene-containing nioplexes were largely internalized via macropinocytosis, exhibited minor lyso- somal 
accumulation and showed high transfection efficiency. On the opposite, macropinocytosis was weakly activated when ARPE- 19 cells were treated with poorly efficient cholesterol-containing nioplexes, which largely accumulated in lysosomes. Aside from clarifying the exact molecular aspects of this correlation, it seems that macropinocytosis could be a preferred internalization route of nioplexe formulations. This observation might explain the superior efficiency of nioplexes prepared with squalene (Fig. 4A bars, Sque) and provides a reasonable explanation for the low transfection efficiency of cholesterol-based nioplexes (Fig. 4A bars, Cho). Notably, this suggestion is in excellent agreement with previous findings (Cardarelli et al., 2012) showing that macropinocytosis is the main pathway of internalization of highly efficient liposome/ DNA complexes. In any case, further experiments with specific inhibitors of the pathways studied such as chlorpromazine, filipin or EIPA (ethylisopropylamiloride) could help us to clarify our hypothesis.

Squalane-containing nioplexes exhibited an endocytic pattern similar to that of nioplexes prepared with squalene. Nevertheless, squalane-containing systems were less efficient (Fig. 4A bars, Squa) than their squalene-enriched counterpart (Fig. 4A bars, Sque). This result was likely due to less involvement of macro- pinocytosis resulting in larger colocalization of complexes with lysosomes (Fig. 6 B). In the absence of helper lipid, nioplexes were poorly efficient (Fig. 4A bars, None), but this was not due to accumulation within acidic lysosomes (Fig. 7, None). These results suggest that despite being able to avoid lysosomal degradation this nioplexe formulation was unable to release their cargo effectively, which can be supported by the agarose gel results, where high DNA condensation was observed (Fig. 3 lane 13).

\section{Conclusion}

In this study, we investigated the role played by the helper lipid on the transfection efficiency of four niosome formulations in ARPE-19 cells. Our results showed that the helper lipid does affect not only to the physico-chemical properties of both bare and DNA- loaded niosomes, but also to important biological processes intimately related with the final transfection efficiency. Collec-tively, our data pointed out that the helper lipid composition has a deep influence on the cellular uptake of nioplexes that, in turn, affects final the fate and transfection efficiency. Niosomes elaborated with squalene as helper lipid showed the highest efficiency to transfect ARPE-19 cells, which could be attributed mainly to their high cellular uptake and posterior entry pathways used, where macropinocytosis pathway and lysosomal elution played an important role. Overall, these studies bring new insights into the role of helper lipids towards the development of highly efficient niosome formulations as non-viral gene delivery vectors for the treatment of inherited retinal diseases. However, we have to consider that both internalization mechanisms and intracellular trafficking processes can be highly modified by interaction of gene carriers with biological fluids, even for retinal application. Therefore, the knowledge of these biological processes that affect to the final transfection efficiency in vivo conditions represents a real challenge for the scientific community in order to design novel gene delivery systems based on non-viral vectors. 


\section{Acknowledgments.}

This project was partially supported by the University of the Basque Country UPV/EHU (UFI 11/32), the National Council of Science and Technology (CONACYT), Mexico, Reg. \# 217101, the Spanish Ministry of Education (Grants CTQ2010-20541, CTQ2010- 14897), the Basque Government (Department of Education, University and Research, predoctoral BFI2011-2226 grant) and by Spanish grants MAT2012-39290-C02-01 and IPT-2012-0574- 300000. Technical and human support provided by SGIker (UPV/ EHU) is gratefully acknowledged. Authors also wish to thank the intellectual and technical assistance from the ICTS "NANBIOSIS", more specifically by the Drug Formulation Unit (U10) of the CIBER in Bioengineering, Biomaterials \& Nanomedicine (CIBER-BBN) at the University of Basque Country (UPV/EHU). GC acknowledges support by the Italian Minister for University and Research (MIUR) (Futuro in Ricerca, Grant No. RBFR08TLPO).

\section{References}

Agirre, M., Ojeda, E., Zarate, J., Puras, G., Grijalvo, S., Eritja, R., García d, C., Barrondo, S., González-Burguera, I., López de, Jesús, Sallés, J., Pedraz, J.L., 2015. New insights into gene delivery to human neuronal precursor NT2Cells: a comparative study between lipoplexes, nioplexes, and polyplexes. Mol. Pharm. 12, 4056-4066.doi:http://dx.doi.org/10.1021/ acs.molpharmaceut.5b00496.

Allain, V., Bourgaux, C., Couvreur, P., 2012. Self-assembled nucleolipids: from supramolecular structure to soft nucleic acid and drug delivery devices. Nucleic Acids Res. 40, 1891-1903. doi:http://dx.doi.org/10.1093/nar/gkr681.

Allison, A.C., Byars, N.E., 1986. An adjuvant formulation that selectively elicits the formation of antibodies of protective isotypes and of cell-mediated immunity. J. Immunol. Methods 95, 157-168.

Anderson, W.F., 1998. Human gene therapy. Nature 392, 25-30. doi:http://dx.doi. org/10.1038/32058.

Bejjani, R.A., BenEzra, D., Cohen, H., Rieger, J., Andrieu, C., Jeanny, J.C., Gollomb, G., BeharCohen, F.F., 2005. Nanoparticles for gene delivery to retinal pigment epithelial cells. Mol. Vis. $11,124-132$.

Byk, G., Dubertret, C., Escriou, V., Frederic, M., Jaslin, G., Rangara, R., Pitard, B., Crouzet, J., Wils, P., Schwartz, B., Scherman, D., 1998. Synthesis activity, and structure-activity relationship studies of novel cationic lipids for DNA transfer. J. Med. Chem. 41, 229-235.

Caracciolo, G., Amenitsch, H., 2012. Cationic liposome/DNA complexes: from structure to interactions with cellular membranes. Eur. Biophys. J. 41, 815-829. doi:http://dx.doi.org/10.1007/s00249-012-0830-8.

Caracciolo, G., Caminiti, R., 2004. DNA-DNA electrostatic interactions within cationic lipid/DNA lamellar complexes. Chem. Phys. Lett. 400, 314-319. doi: http://dx.doi.org/10.1016/j.cplett.2004.10.134. 
Caracciolo, G., Caminiti, R., Digman, M.A., Gratton, E., Sanchez, S., 2009. Efficient escape from endosomes determines the superior efficiency of multicomponent lipoplexes. J. Phys. Chem. B 113, 4995-4997. doi:http://dx.doi.org/10.1021/jp811423r.

Cardarelli, F., Pozzi, D., Bifone, A., Marchini, C., Caracciolo, G., 2012. Cholesterol- dependent macropinocytosis and endosomal escape control the transfection efficiency of lipoplexes in CHO living cells. Mol. Pharm. 9, 334-340. doi:http:// dx.doi.org/10.1021/mp200374e.

Charbel Issa, P., MacLaren, R.E., 2012. Non-viral retinal gene therapy: a review. Clin. Exp. Ophthalmol. 40, 39-47. doi:http://dx.doi.org/10.1111/j.1442- 9071.2011.02649.x.

Choi, W.J., Kim, J.K., Choi, S.H., Park, J.S., Ahn, W.S., Kim, C.K., 2004. Low toxicity of cationic lipid-based emulsion for gene transfer. Biomaterials 25, 5893-5903. doi:http://dx.doi.org/10.1016/j.biomaterials.2004.01.031.

Chung, H., Kim, T.W., Kwon, M., Kwon, I.C., Jeong, S.Y., 2001. Oil components modulate physical characteristics and function of the natural oil emulsions as drug or gene delivery system. J. Control. Release 71, 339-350. doi:http://dx.doi. org/10.1016/S0168-3659(00) 00363-1.

Dabkowska, A.P., Barlow, D.J., Campbell, R.A., Hughes, A.V., Quinn, P.J., Lawrence, M. J., 2012. Effect of helper lipids on the interaction of DNA with cationic lipid monolayers studied by specular neutron reflection. Biomacromolecules 13, 2391-2401. doi: 10.1021/bm300639n.

Gillard, M., Jia, Z., Hou, J.J., Song, M., Gray, P.P., Munro, T.P., Monteiro, M.J., 2014. Intracellular trafficking pathways for nuclear delivery of plasmid DNA complexed with highly efficient endosome escape polymers. Biomacromolecules 15, 3569-3576. doi: $10.1021 / \mathrm{bm} 5008376$.

Gratton, S.E., Ropp, P.A., Pohlhaus, P.D., Luft, J.C., Madden, V.J., Napier, M.E., DeSimone, J.M., 2008. The effect of particle design on cellular internalization pathways. Proc. Natl. Acad. Sci. U. S. A. 105, 11613-11618. doi: 10.1073/pnas.0801763105.

Huang, Y., Rao, Y., Chen, J., Yang, V.C., Liang, W., 2011. Polysorbate cationic synthetic vesicle for gene delivery. J. Biomed. Mater. Res. A. 96, 513-519. doi: 10.1002/jbm.a.32999.

Jayaraman, M., Ansell, S.M., Mui, B.L., Tam, Y.K., Chen, J., Du, X., Butler, D., Eltepu, L., Matsuda, S., Narayanannair, J.K., Rajeev, K.G., Hafez, I.M., Akinc, A., Maier, M.A., Tracy, M.A., Cullis, P.R., Madden, T.D., Manoharan, M., Hope, M.J., 2012. Maximizing the potency of siRNA lipid nanoparticles for hepatic gene silencing in vivo. Angew. Chem. Int. Ed. Engl. 51, 85298533. doi: 10.1002/anie.201203263.

Karmali, P.P., Chaudhuri, A., 2007. Cationic liposomes as non-viral carriers of gene medicines: resolved issues, open questions, and future promises. Med. Res. Rev. 27, 696-722. doi: 10.1002/med.20090.

Kim, Y.J., Kim, T.W., Chung, H., Kwon, I.C., Sung, H.C., Jeong, S.Y., 2003. The effects of serum on the stability and the transfection activity of the cationic lipid emulsion with various oils. Int. J. Pharm. 252, 241-252. 
Kiss, A.L., Botos, E., 2009. Endocytosis via caveolae: alternative pathway with distinct cellular compartments to avoid lysosomal degradation? J. Cell. Mol. Med. 13, 1228-1237. doi: 10.1111/j.1582-4934.2009.00754.x.

Koivisto, P.V., Miettinen, T.A., 1988. Increased amounts of cholesterol precursors in lipoproteins after ileal exclusion. Lipids 23, 993-996. Lim, J.P., Gleeson, P.A., 2011. Macropinocytosis: an endocytic pathway for internalising large gulps. Immunol. Cell Biol. 89, 836-843. doi: 10.1038/icb.2011.20.

Liu, G.C., Ahrens Jr., E.H., Schreibman, P.H., Crouse, J.R., 1976. Measurement of squalene in human tissues and plasma: validation and application. J. Lipid Res. 17, 38-45.

Luzio, J.P., Parkinson, M.D., Gray, S.R., Bright, N.A., 2009. The delivery of endocytosed cargo to Iysosomes. Biochem. Soc. Trans. 37, 1019-1021. doi: 10.1042/BST0371019.

Marchini, C., Pozzi, D., Montani, M., Alfonsi, C., Amici, A., Amenitsch, H., Candeloro De Sanctis, S., Caracciolo, G., 2010. Tailoring lipoplex composition to the lipid composition of plasma membrane: a Trojan horse for cell entry? Langmuir 26, 13867-13873. doi: 10.1021/la1023899.

Martin, B., Sainlos, M., Aissaoui, A., Oudrhiri, N., Hauchecorne, M., Vigneron, J.P., Lehn, J.M., Lehn, P., 2005. The design of cationic lipids for gene delivery. Curr. Pharm. Des. 11, 375-394.

Midoux, P., Monsigny, M., 1999. Efficient gene transfer by histidylated polylysine/ pDNA complexes. Bioconjug. Chem. 10, 406-411. doi: 10.1021/ bc9801070.

Mochizuki, S., Kanegae, N., Nishina, K., Kamikawa, Y., Koiwai, K., Masunaga, H., Sakurai, K., 2013. The role of the helper lipid dioleoylphosphatidylethanolamine (DOPE) for DNA transfection cooperating with a cationic lipid bearing ethylenediamine. Biochim. Biophys. Acta 1828, 412-418. doi: 10.1016/j.bbamem.2012.10.017.

Moghassemi, S., Hadjizadeh, A., 2014. Nano-niosomes as nanoscale drug delivery systems: an illustrated review. J. Control. Release 185, 22-36. doi: 10.1016/j.jconrel.2014.04.015.

Ochoa, G.P., Sesma, J.Z., Diez, M.A., Diaz-Tahoces, A., Aviles-Trigeros, M., Grijalvo, S., Eritja, R., Fernandez, E., Pedraz, J.L., 2014. A novel formulation based on 2,3-di (tetradecyloxy)propan-1amine cationic lipid combined with polysorbate 80 for efficient gene delivery to the retina. Pharm. Res. 31, 1665-1675. doi: 10.1007/s11095-013-1271-5.

Ojeda, E., Puras, G., Agirre, M., Zarate, J., Grijalvo, S., Eritja, R., Martinez-Navarrete, G., SotoSanchez, C., Diaz-Tahoces, A., Aviles-Trigueros, M., Fernandez, E., Pedraz, J. L., 2015a. The influence of the polar head-group of synthetic cationic lipids on the transfection efficiency mediated by niosomes in rat retina and brain. Biomaterials 77, 267-279 S0142-9612(15) 00913-8[pii].

Ojeda, E., Puras, G., Agirre, M., Zarate, J., Grijalvo, S., Pons, R., Eritja, R., Martinez- Navarrete, G., Soto-Sanchez, C., Fernandez, E., Pedraz, J.L., 2015b. Niosomes based on synthetic cationic lipids for gene delivery: the influence of polar head-groups on the transfection efficiency in HEK-293, ARPE-19 and MSC-D1 cells. Org. Biomol. Chem. 13, 1068-1081. doi: 10.1039/c4ob02087a. 
Pozzi, D., Marchini, C., Cardarelli, F., Amenitsch, H., Garulli, C., Bifone, A., Caracciolo, G., 2012. Transfection efficiency boost of cholesterol-containing lipoplexes. Biochim. Biophys. Acta 1818, 2335-2343. doi: 10.1016/j. bbamem.2012.05.017.

Pozzi, D., Marchini, C., Cardarelli, F., Salomone, F., Coppola, S., Montani, M., Zabaleta, M.E., Digman, M.A., Gratton, E., Colapicchioni, V., Caracciolo, G., 2014. Mechanistic evaluation of the transfection barriers involved in lipid-mediated gene delivery: interplay between nanostructure and composition. Biochim. Biophys. Acta 1838, 957-967. doi: 10.1016/j. bbamem.2013.11.014.

Puras, G., Zarate, J., Diaz-Tahoces, A., Aviles-Trigueros, M., Fernandez, E., Pedraz, J.L., 2013. Oligochitosan polyplexes as carriers for retinal gene delivery. Eur. J. Pharm. Sci. 48, 323-331. doi: 10.1016/j.ejps.2012.11.009.

Puras, G., Mashal, M., Zarate, J., Agirre, M., Ojeda, E., Grijalvo, S., Eritja, R., Diaz- Tahoces, A., Martinez Navarrete, G., Aviles-Trigueros, M., Fernandez, E., Pedraz, J. L., 2014. A novel cationic niosome formulation for gene delivery to the retina. J. Control. Release 174, 27-36. doi: 10.1016/j.jconrel.2013.11.004.

Reddy, L.H., Couvreur, P., 2009. Squalene: a natural triterpene for use in disease management and therapy. Adv. Drug Deliv. Rev. 61, 1412-1426. doi: 10.1016/j.addr.2009.09.005.

Rejman, J., Oberle, V., Zuhorn, I.S., Hoekstra, D., 2004. Size-dependent internalization of particles via the pathways of clathrin- and caveolae-mediated endocytosis. Biochem. J. 377, 159-169. doi: 10.1042/ BJ20031253.

Rezvani Amin, Z., Rahimizadeh, M., Eshghi, H., Dehshahri, A., Ramezani, M., 2013. The effect of cationic charge density change on transfection efficiency of polyethylenimine. Iran. J. Basic Med. Sci. 16, 150-156.

Semple, S.C., Akinc, A., Chen, J., Sandhu, A.P., Mui, B.L., Cho, C.K., Sah, D.W., Stebbing, D., Crosley, E.J., Yaworski, E., Hafez, I.M., Dorkin, J.R., Qin, J., Lam, K., Rajeev, K.G., Wong, K.F., Jeffs, L.B., Nechev, L., Eisenhardt, M.L., Jayaraman, M., Kazem, M., Maier, M.A., Srinivasulu, M., Weinstein, M.J., Chen, Q., Alvarez, R., Barros, S.A., De, S., Klimuk, S.K., Borland, T., Kosovrasti, V., Cantley, W.L., Tam, Y.K., Manoharan, M., Ciufolini, M.A., Tracy, M.A., de Fougerolles, A., MacLachlan, I., Cullis, P.R., Madden, T.D., Hope, M.J., 2010. Rational design of cationic lipids for siRNA delivery. Nat. Biotechnol. 28, 172-176. doi: 10.1038/nbt.1602.

E. Ojeda et al. / International Journal of Pharmaceutics 503 (2016) 115-126 125Seubert, A., Monaci, E., Pizza, M., O'Hagan, D.T., Wack, A., 2008. The adjuvants aluminum hydroxide and MF59 induce monocyte and granulocyte chemoattractants and enhance monocyte differentiation toward dendritic cells. J. Immunol. 180, 5402-5412 180/8/5402[pii].

Stellwagen, N.C., Stellwagen, E., 2009. Effect of the matrix on DNA electrophoretic mobility. J. Chromatogr. A 1216, 1917-1929. doi:http://dx.doi.org/10.1016/j. chroma.2008.11.090. Stewart, M.E., 1992. Sebaceous gland lipids. Semin. Dermatol. 11, 100-105. 
Xiang, S., Tong, H., Shi, Q., Fernandes, J.C., Jin, T., Dai, K., Zhang, X., 2012. Uptake mechanisms of non-viral gene delivery. J. Control. Release 158, 371-378. doi: 10.1016/j.jconrel.2011.09.093.

Zhao, F., Zhao, Y., Liu, Y., Chang, X., Chen, C., Zhao, Y., 2011. Cellular uptake, intracellular trafficking, and cytotoxicity of nanomaterials. Small 7, 1322-1337. doi: 10.1002/smll.201100001.

Zhi, D., Zhang, S., Wang, B., Zhao, Y., Yang, B., Yu, S., 2010. Transfection efficiency of cationic lipids with different hydrophobic domains in gene delivery. Bioconjug. Chem. 21, 563-577. doi: 10.1021/bc900393r.

Zuhorn, I.S., Bakowsky, U., Polushkin, E., Visser, W.H., Stuart, M.C., Engberts, J.B., Hoekstra, D., 2005. Nonbilayer phase of lipoplex-membrane mixture determines endosomal escape of genetic cargo and transfection efficiency. Mol. Ther. 11, 801-810 S1525-0016(04) 01595-3[pii].

dos Santos, T., Varela, J., Lynch, I., Salvati, A., Dawson, K.A., 2011. Quantitative assessment of the comparative nanoparticle-uptake efficiency of a range of cell lines. Small 7, 3341-3349. doi: 10.1002/smll.201101076. 


\section{Supporting Information}

Synthesis of the ionizable cationic amino lipid. All reactions were carried out under an inert atmosphere of argon. Flash column chromatography was performed on silica gel SDS 0.063-0.2 mm/70-230 mesh. ${ }^{1} \mathrm{H}$ and ${ }^{13} \mathrm{C}$ NMR spectra were recorded at $25{ }^{\circ} \mathrm{C}$ on a Varian Mercury $400 \mathrm{MHz}$ spectrometer using deuterated solvents. Tetramethylsilane (TMS) was used as an internal reference $(0 \mathrm{ppm})$ for ${ }^{1} \mathrm{H}$ spectra recorded in $\mathrm{CDCl}_{3}$ and the residual signal of the solvent $(77.1 \mathrm{ppm})$ for ${ }^{13} \mathrm{C}$ spectra. Chemical shifts are reported in parts per million (ppm), coupling constants ( $\mathrm{J}$ ) in $\mathrm{Hz}$ and multiplicity as follows: $\mathrm{s}$ (singlet), $\mathrm{t}$ (triplet), and $\mathrm{m}$ (multiplet). Electrospray ionization mass spectra (ESI-MS) were recorded on a high resolution ESI-MS measured on an Agilent 1100 LC/MS-TOF instrument (Servei d'Espectrometría de Masses, Universitat de Barcelona).

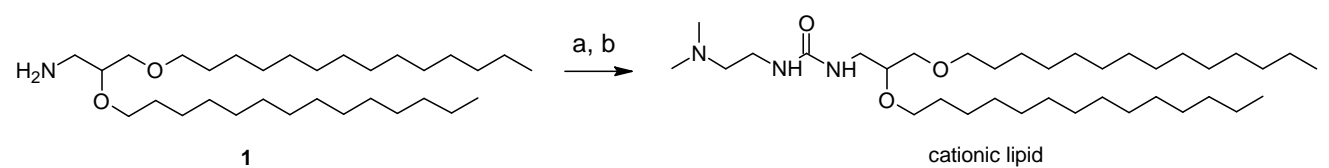

Reagents and Conditions a, p-nitrophenyl chloroformate, DIEA, TFH:DCM (1:1), 4h, r.t.; b. 1, DMF, overnight, r.t.

Figure SI 1. Synthesis of cationic lipid 1-(2-dimethylaminoethyl)-3-[2,3di(tetradecoxy)propyl]urea. Amino lipid (1) (100 mg; $0.206 \mathrm{mmol}$ ) and p-nitrophenylchloroformate (2.5 eq) were dissolved in a mixture of tetrahydrofurane: dichloromethane (1:1) (6 mL). The reaction was cooled at $0{ }^{\circ} \mathrm{C}$, and DIEA (2.5 eq) was carefully added dropwise. The solution was stirred for 4 hours at room temperature. The solvent was removed in vacuo, and the resultant crude was used in the next step without further purification. The yellow crude was dissolved in dimethylformamide (DMF) $(3 \mathrm{~mL})$, and the corresponding dimethylamine derivative (1.1 eq) was added dropwise. The reaction was stirred overnight at room temperature. Finally, DMF was removed under vacuum, and the resultant crude was purified by flash 
chromatography (DCM: MeOH 5\% to 10\%). Yield 65\%; ${ }^{1} \mathrm{H}$-NMR (400 MHz, $\left.\mathrm{CDCl}_{3}\right) \square 3.44$ (m, 6H; $\left.3 \mathrm{CH}_{2}-\mathrm{O}\right) ; 3.26$ (m, 3H; CH-N and $\left.\mathrm{CH}_{2}-\mathrm{O}\right) ; 2.46$ (m, 4H; N- $\left.\mathrm{CH}_{2}-\mathrm{CH}_{2}-\mathrm{N}\right), 2.28$ (s, 3H; $\left.\mathrm{N}-\mathrm{CH}_{3}\right), 2.25\left(\mathrm{~s}, 3 \mathrm{H} ; \mathrm{N}-\mathrm{CH}_{3}\right), 1.55\left(\mathrm{~m}, .2 \mathrm{H} ; \mathrm{CH}_{2}-\mathrm{CH} 2\right), 1.25$ (m, 46H; alkyl chain); 0.88 (t, $J=$ $\left.6.7 \mathrm{~Hz} ; 6 \mathrm{H} ; 2 \mathrm{CH}_{3}\right) ;{ }^{13} \mathrm{C}-\mathrm{NMR}\left(125 \mathrm{MHz}, \mathrm{CDCl}_{3} \square 161.1(\mathrm{CO}) ; 158.6(\mathrm{CO}), 71.7\left(\mathrm{CH}_{2}-\mathrm{O}\right)\right.$, $70.2\left(\mathrm{CH}_{2}-\mathrm{O}\right), 58.8\left(\mathrm{CH}_{2}-\mathrm{O}\right), 57.5(\mathrm{CH}-\mathrm{O}), 45.0\left(\mathrm{CH}_{3}-\mathrm{N}\right), 41.9\left(\mathrm{CO}-\mathrm{CH}_{2}-\mathrm{N}\right), 37.7\left(\mathrm{CH}_{2}-\mathrm{N}\right)$, $35.2\left(\mathrm{CH}_{2}-\mathrm{N}\right), 31.8\left(\mathrm{CH}_{2}-\mathrm{N}\right), 30.0,29.7,29.6,29.4,29.3,26.1,22.6$ (alkyl chain), $14.0\left(\mathrm{CH}_{3}\right)$; ESI-MS for $\mathrm{C}_{31} \mathrm{H}_{65} \mathrm{NO}_{2} \mathrm{~m} / \mathrm{z} 598.5808$ (calculated) $598.5806(\mathrm{M}+\mathrm{H})^{+}$(found).

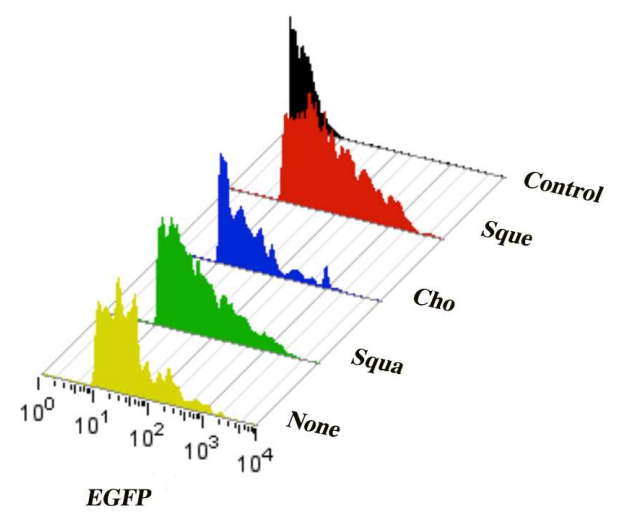

Figure SI 2. Flow cytometry histograms of transfected cells with nioplexes prepared with different helper lipids in ARPE-19 cells. Color code: Black) control cells, Red) nioplexes prepared with squalene (Sque), Blue) nioplexes prepared with cholesterol (Cho), Green) nioplexes prepared with squalane (Squa) and Yellow) nioplexes prepared without helper lipid (None). (For interpretation of the references to color in this figure legend, the reader is referred to the web version of the article).

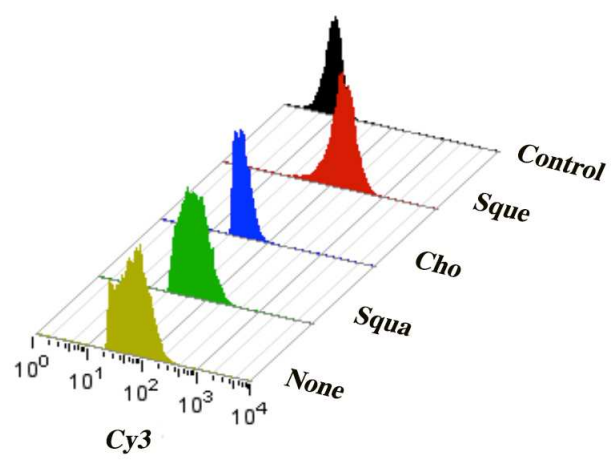


Figure SI 3. Flow cytometry histograms of cell uptake with nioplexes prepared with different helper lipids in ARPE-19 cells. Color code: Black) control cells, Red) nioplexes prepared with squalene (Sque), Blue) nioplexes prepared with cholesterol (Cho), Green) nioplexes prepared with squalane (Squa) and Yellow) nioplexes prepared without helper lipid (None). (For interpretation of the references to color in this figure legend, the reader is referred to the web version of the article).

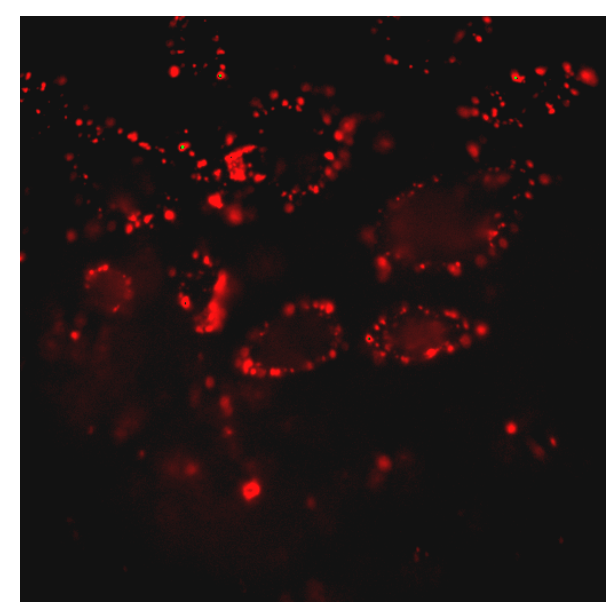

Figure SI 4. Confocal image of the ARPE-19 cells in contact with nioplexes based on squalene at 5 min. The stained DNA-niosome complex is shown in red. For interpretation of the referenced color in this figure legend, the reader is referred to the web version of the article. 